\title{
Impact of Pressure Ulcers on Therapeutic Outcomes in Inpatient Physiotherapy Services
}

\author{
Sandeep Babasaheb Shinde ${ }^{1}$, G. Varadharajulu² \\ ${ }^{1}$ Department of Physiotherapy, Krishna Institute of Medical Sciences Deemed to be \\ University, Karad, Maharashtra, India. ${ }^{2}$ Department of Physiotherapy, Krishna Institute of \\ Medical Sciences Deemed to be University, Karad, Maharashtra, India.
}

\section{ABSTRACT}

\section{BACKGROUND}

Pressure ulcers have been associated with an extended length of hospitalization, sepsis and mortality. Over the last few decades, little has been written about the impact of pressure ulcers on therapeutic outcomes in inpatient physiotherapy services. It is obvious that critically ill patients, who are sedated, ventilated, patients with chronic neurological, musculoskeletal and cardio-respiratory disorders who are bed ridden for prolonged period of time are particularly at risk of developing skin breakdown. This will help in identifying barriers to patient participation and develop strategies to pressure ulcer prevention. We wanted to determine the impact of pressure ulcers on therapeutic outcomes in inpatient physiotherapy services.

\section{METHODS}

The study was conducted from June 2015 to January 2016. The impact of pressure ulcer on inpatient physiotherapy services was determined by detailed physical assessment, evaluation with Functional Independence Measure score (FIM) and Braden Q Scale. A total 96 subjects were assessed during this time.

\section{RESULTS}

There was statistically significant impact of pressure ulcers on therapeutic outcomes in inpatient physiotherapy services. The FIM takes into consideration self-care, sphincter control, transfers, locomotion, communication social cognition. A poor score denotes poor outcome at discharge. Tissue level assessment was carried out by using Braden Q Scale, a poor score of which again denotes very poor outcome in mobility, activity, sensory perception, moisture, friction - shear, nutrition, tissue perfusion and oxygenation.

\section{CONCLUSIONS}

Pressure ulcer is one of the preventable problems; but in majority of cases it becomes the main reason for poor therapeutic outcome.

\section{KEY WORDS}

Pressure Ulcer, Functional Mobility, Functional Independence Measure Score, Braden Q Scale, Therapeutic Outcome
Corresponding Author: Dr. Sandeep Babasaheb Shinde, Associate Professor,

Faculty of Physiotherapy, Krishna Institute of Medical Sciences, Deemed to be University, Karad, Maharashtra, India. E-mail: drsandeepshinde24@gmail.com

DOI: $10.14260 / j e m d s / 2020 / 476$

How to Cite This Article:

Shinde SB, Varadharajulu G. Impact of pressure ulcers on therapeutic outcomes in inpatient physiotherapy services. J. Evolution Med. Dent. Sci. 2020;9(31): 21872190, DOI: 10.14260/jemds/2020/476

Submission 01-04-2020,

Peer Review 26-06-2020,

Acceptance 03-07-2020,

Published 03-08-2020.

Copyright (C) 2020 JEMDS. This is an open access article distributed under Creative Commons Attribution License [Attribution 4.0 International (CC BY 4.0)] 


\section{BACKGROUND}

Pressure ulcer is a preventable cause of multiple complications leading to worsening of health condition and even death. Pressure ulcers are often undertreated. A pressure ulcer is a break in the integument usually caused by continuous pressure to skin and muscle. ${ }^{1}$ Although these ulcers can occur anywhere on the body, they are often located in the trochanteric, ischial, heel, and sacral areas. Patients may not immediately be aware of these developing wounds, because they often occur in bed-bound, paralyzed, and elderly patients undergoing treatment for other diseases. The prevalence of pressure ulcer is increasing in Indian health care settings. This condition leads to great economic burden on patient and his family. Government agencies involved in accreditation of quality health care gives higher grading for prevention of the pressure ulcer. Immobility is greatest risk factor for development of pressure ulcer. ${ }^{2}$ Physiotherapy interventions such as relaxed passive movements, positioning and bed mobility measures are traditionally used for the prevention of pressure ulcers. Patients have higher amount of loading and weight distribution on bony prominences such as external occipital protuberance, spine of scapulae, $\mathbf{c}_{7}$ vertebra, iliac crest, gluteal region and calcaneum etc. These areas of higher amount of loading and weight distribution and are the common sites for the development of pressure ulcers. ${ }^{3}$ For physiotherapy interventions the handling skills used for the training the bed mobility and functional mobility requires handling majority of these pressure areas. Hand placements during the training of bed mobility can be disturbed by pressure ulcers. The surface anatomical landmarks for the proximal key points are posterior aspect of scapulae and distal key points are the iliac crest are important for training of activities such as supine lying to rolling to one side, side lying to sitting and sitting to standing. Pressure ulcer can affect implementation of various physiotherapy interventions ${ }^{4}$. If mobility measures for Comatose and Neurological patients with serious involvement of brain and spinal cord are insufficient then complications such as joint contractures and pressure ulcers are very common. Identification of barriers for patient handling is essential to reform the physiotherapy strategies.

Poor adherence to treatment and follow up can have negative effects on therapeutic outcomes and healthcare cost. However, very little is known about the barriers to treatment adherence within physiotherapy. In this study efforts were made to identify barriers to treatment adherence in chronic conditions typically managed in tertiary care hospital. There was strong evidence that poor treatment adherence was associated with low levels of physical activity at baseline or in previous weeks, low in-treatment adherence with exercise, low self-efficacy, depression, anxiety, helplessness, poor social support/activity, greater perceived number of barriers to exercise and increased pain levels during exercise ${ }^{5,6}$. Strategies to overcome these barriers and improve adherence are need to find out. Much of the available research has focused on patient factors and additional research is required to investigate the barriers introduced by health procedures, standard protocols of health organizations Members of Health delivery team etc. Since these factors are also likely to influence patient adherence with treatment.
Identification of these barriers during patient evaluation may be important in order to plan specific management strategies which help to counteract effects of pressure ulcer and improve therapeutic outcome. Physiotherapists should be concerned about the barriers facing their patients and act comprehensively with their patients to plan suitable treatment plans which are customized to the patient's problems. There was conflicting evidence for age, nature of illness and pain. Very little evidence was available for many other barriers. In addition there is a lack of research investigating barriers introduced by health care professionals, hospitals and organizations. More high quality research is required to increase our understanding of all the factors which contribute to barriers for physiotherapy intervention. Barriers to early mobilization of ICU patients are multifactorial, with patient related barriers being most commonly mentioned. Studies have identified numerous practical strategies that have been successful in addressing most perceived barriers to early mobilization. Systematic efforts to change ICU culture to prioritize early mobilization using an interprofessional and patient centered approach and multiple targeted strategies are important components of successfully implementing early mobility in clinical practice. In many hospital set ups mere barrier for mobility at the end proves to be fatal. The barriers for physiotherapy because of pressure ulcer can lead to worsening to physical functioning of patients. Lack of mobility can lead to formation deep vein thrombosis, embolism in certain high risk patients. Peripheral vascular disease severity gets increased with pronged bed ridden state. ${ }^{7}$ Secondary manifestations of prolonged bed ridden stage are muscle weakness, muscle atrophy; muscle tightness, joint stiffness, postural faults and sometimes joint deformities may become irreversible.7,8,9 Pressure ulcer is one of the preventable problem but in majority of cases it becomes main reason for poor therapeutic outcome. In current practice with significant modernization and use of technologies for nursing care threat is always inevitable if any patient develops pressure ulcer.

Pressure ulcers causes financial crisis on patient and agencies who pay for health care costs. Chronic ulcers in patients with serious illnesses are many times not given priority. This allows to ulcer progression because the main aim of hospitalization is on the illness on admission. Healthcare workers, patient, caretakers and patient's family members must be trained to detect these ulcers early in order to prevent progression to more advanced stages. Regular observation should continue at home. Patients need to be trained to examine themselves with proper palpation and observation mirrors to detect newly developing pressure ulcers.

\section{METHODS}

We conducted this observational study in Krishna Hospital and research centre Karad. The total 96 in-patients were recruited by using systematic random sampling and they were assessed for 6 months. Previous studies Ampiah PK, Ahenkorah J Shown 120 participants. ${ }^{10}$ Minimum subjects required with $95 \%$ confidence and $80 \%$ power is $n=90$, since follow up study, $\mathrm{n}=21+3$ (since $10 \%$ attrition rate) $=23$ Hence minimum 23 subjects in either group should be studied. In this study patients were randomly allocated by using random allocation software in one group of 96 . Both the genders were 
included. Inclusion criteria for the study was Patients with the pressure ulcers those are present on admission to Hospital. Patients with the pressure ulcers those are developed during hospital stay. All the preoperative and postoperative Patients. All critically ill patients, who are sedated and ventilated. All patients with Chronic Neurological, Musculoskeletal and Cardio-Respiratory Disorders that are bed ridden for prolonged period of time. The musculoskeletal in-patients with multiple traumatic injuries such as spinal and extremity fractures, open wounds, patients with external fixators, patient with injuries to thorax and visceral organs were assessed. The neurological in-patients with stroke, traumatic brain injury, spinal cord injury and prolonged bed ridden patients were assessed. The Cardio-respiratory in-patients with acute respiratory distress syndrome, metabolic encephalopathy were assessed. The patients with skin conditions Burns Psoriasis Herpes zoster etc., were excluded. The assessment of selected patients was done at the time of admission and discharge from the hospital. Many patients were found with pressure ulcer on admission itself as they were transferred from higher centers to our tertiary care hospital. Patients were treated for chronic illness such as spinal cord injury, stroke, head injury, multiple fractures in higher centers and then they were transferred to tertiary care hospital for further medical and rehabilitative care. The outcome measured used to determine functional mobility were detailed Physical assessment, with Functional independence measure score (FIM) and Braden Q Scale. The assessment findings were documented and analyzed.11,12

\section{Statistical Analysis}

Within Group Comparisons: Within group comparison was done by applying Paired $t$ test to pre and post values of same group for all the outcome measures. Descriptive statistical measures were applied Measures of central tendency and measures of variability (spread).

\section{RESULTS}

\begin{tabular}{|c|c|c|c|}
\hline Sr. No. & Demograp & $\mathbf{i a b}$ & \\
\hline & Present $(\mathrm{N}) \mathrm{I}$ & $\operatorname{lge}\left({ }^{\circ}\right.$ & \\
\hline 1. & & ars) & \\
\hline & $20-50$ & 38 & 39.58 \\
\hline & $51-80$ & 58 & 60.41 \\
\hline 2. & & & \\
\hline & Male & 52 & 54.16 \\
\hline & Female & 44 & 45.83 \\
\hline 3. & & oitat & \\
\hline & Rural & 71 & 73.95 \\
\hline & Urban & 25 & 26.04 \\
\hline 4. & & cory & \\
\hline & Diabetes Mellitus & 69 & 71.87 \\
\hline & Neurological Problems & 56 & 58.33 \\
\hline & Hypertension & 61 & 63.54 \\
\hline 5. & Trea & Histo & \\
\hline & Operative & 23 & 23.95 \\
\hline & Conservative treatment & 73 & 76.04 \\
\hline 6. & Obesity & 81 & 84.37 \\
\hline & Non Obese & 15 & 15.62 \\
\hline 7. & Duration of bed ridden stage & & \\
\hline & Less than 3 weeks & 6 & 0.062 \\
\hline & 3 weeks to 3 months & 44 & 45.83 \\
\hline & More than 3 months & 46 & 47.91 \\
\hline
\end{tabular}

Study population was mainly rural patients (73.95\%), above 50 years of age (60.41\%), Diabetes mellitus (71.87\%) Hypertension (58.33\%), Postoperative (23.95\%) conservatively managed (76.04\%), and Obese (84.37\%).

\begin{tabular}{|ccccccc|}
\hline Sr. & Item & \multicolumn{2}{c|}{ Admission } & \multicolumn{2}{c|}{ Discharge } & P Value \\
No. & Mean & S.D. & Mean & S.D. & \\
1 & Self-Care & 1.46 & 1.03 & 1.33 & 0.98 & 0.0699 \\
2 & Sphincter Control & 1.18 & 0.91 & 1.01 & 0.93 & 0.0741 \\
3 & Transfers & 1.23 & 1.04 & 2.08 & 1.59 & 0.0543 \\
4 & Locomotion & 1.42 & 1.01 & 2.24 & 1.83 & 0.861 \\
5 & Communication & 3.22 & 2.51 & 5.14 & 3.63 & 0.0231 \\
6 & Social Cognition & 3.41 & 2.30 & 5.21 & 3.76 & 0.642 \\
\hline \multicolumn{7}{|c|}{ Table 2. Impact of Pressure Ulcers on Functional Mobility in } \\
Inpatient Physiotherapy Services: Functional Independence \\
Measure Score (FIM)
\end{tabular}

Functional mobility in inpatient physiotherapy services. All the items Self-Care, Sphincter Control, Transfers, Locomotion, Communication Social Cognition were denoting poor outcome at discharge.

\begin{tabular}{|ccccccc|}
\hline Sr. & Item & \multicolumn{2}{c|}{ Admission } & \multicolumn{2}{c|}{ Discharge } & P Value \\
No. & & Mean & S.D. & Mean & S.D. & \\
1 & Mobility & 1.08 & 0.81 & 1.23 & 1.06 & 0.0621 \\
2 & Activity & 1.02 & 0.89 & 1.11 & 0.92 & 0.0762 \\
3 & Sensory Perception & 1.89 & 1.41 & 1.45 & 0.71 & 0.0847 \\
4 & Moisture & 2.39 & 1.86 & 2.89 & 0.94 & 0.0545 \\
5 & Friction - Shear & 1.91 & 1.46 & 1.86 & 0.76 & 0.654 \\
6 & Nutrition & 2.76 & 2.11 & 3.56 & 1.74 & 0.587 \\
7 & Tissue Perfusion and & 2.27 & 1.71 & 2.33 & 1.04 & 0.754 \\
\hline Oxygenation & Table 3. Impact of Pressure Ulcers on Functional Mobility in \\
\hline Inpatient Physiotherapy Services: Braden Q Scale \\
\hline
\end{tabular}

All items in Mobility, Activity, Sensory Perception, Moisture, Friction - Shear, Nutrition, Tissue Perfusion and Oxygenation were denoting poor outcome at discharge.

\section{DISCUSSION}

In this article, we outline an example how the pressure ulcers can negatively affect the therapeutic outcomes in inpatient physiotherapy services. Healthcare workers are often hesitant to diagnose pressure ulcers for fear of being blamed for ulcer development and because Medico-legal interventions and patient's rights are increasingly demanding accountability for healthcare. Although healthcare workers must be accountable for their actions, contributing factors such as advanced age, prolonged immobility, and frailty also must be taken into consideration when incidence is calculated and analysed. As the geriatric population continues to grow at significant rate across the globe, there will inevitably be more chronically and critically ill patients in the healthcare system. ${ }^{13}$ Because these populations are at higher risk of developing pressure ulcers, the establishment of a comprehensive protocol for treatment of pressure ulcers is needed. Whereas prevention remains the ideal goal. Our study emphasized detection actual therapeutic outcomes in presence of pressure ulcer.

In this study the therapeutic outcomes were considerably poor for these patients. The main reason behind this issue is pressure ulcer. Study population was mainly rural patients (73.95\%), above 50 years of age $(60.41 \%)$, Diabetes mellitus (71.87\%) Hypertension (58.33\%), Postoperative (23.95\%) conservatively managed $(76.04 \%)$, and Obese $(84.37 \%)$. The pressure ulcer contributed for development of Fever, Malaise, and systemic problems. This resulted in postponing of 
physiotherapy sessions. Many patients were not received Physiotherapy sessions regularly. This resulted in decreased bed mobility, Restricted joint range of motion, Multiple joint pain, Dependent oedema, Muscle tightness joint capsular contractures, Muscle atrophy and muscle weakness. All these deleterious effects are assessed by Functional independence measure score (FIM). ${ }^{11}$ It helped to determine Impact of pressure ulcers on functional mobility in inpatient physiotherapy services. All the items Self-Care, Sphincter Control, Transfers, Locomotion, Communication Social Cognition were denoting poor outcome at discharge. The more details at tissue level assessment was carried out by using Braden Q Scale, it denotes again very poor outcome in Mobility, Activity, Sensory Perception, Moisture, Friction - Shear, Nutrition, Tissue Perfusion and Oxygenation. ${ }^{12}$

Patients were treated for chronic illness such as spinal cord injury, stroke, head injury, multiple fractures in higher centers and then they were transferred to tertiary care hospital for further medical and rehabilitative care. Now it is bound duty of health care providers of tertiary care hospital to provide quality health care and rehabilitative measures for better patient outcome. The entire health care team is responsible for therapeutic outcome of individual patient in the context of primary disease condition. In tertiary care hospital settings numerous factors are reported which influences the final therapeutic outcome. Pressure ulcer is very much preventable problem and if not prevented it can worsen patient condition, it prolongs patient hospital stay and definitely this is single and most common factor with enormous negative impact on therapeutic outcome. Physiotherapists many times finds very difficult to handle cases with pressure ulcer. If Pressure ulcers are multiple then it becomes real challenge to give routine physiotherapy protocols. Multiple pressure sore limit patients bed mobility and this will result into more and more secondary complications of bed rest. ${ }^{14}$ Efforts are required to prevent formation of pressure sore and in cases with already suffering from pressure sores modified and timely bed mobility regimens to prevent secondary complications. Team efforts are must to deliver quality care and for better therapeutic outcome.

\section{CONCLUSIONS}

Pressure ulcer is one of the preventable problems; but in majority of cases it becomes the main reason for poor therapeutic outcome. In current practice, with significant modernization and use of technologies for nursing care, threat is inevitable if a patient develops pressure ulcer.

This study was approved by the institutional committee of KIMSDU Karad.

\section{Source of Funding}

Dr. Shinde reports grants from Krishna Institute of Medical Sciences, Deemed to be University, Karad. outside the submitted work. Dr. Varadharajulu reports grants from Krishna Institute of Medical Sciences, Deemed to be University, Karad. outside the submitted work.

\section{REFERENCES}

[1] Peterson JZ. safety issues with the elderly and chronically ill. In: Byers JF, White SV, eds. Patient Safety: principles and practice. New York: Springer 2004: p. 421.

[2] Lyder CH, Ayello EA. Pressure ulcers: a patient safety issue. In: Hughes RG, ed. Patient safety and quality: an evidence-based handbook for nurses. Rockville (MD): Agency for Healthcare Research and Quality (US) 2008: p. 12.

[3] Mimura M, Ohura T, Takahashi M, et al. Mechanism leading to the development of pressure ulcers based on shear force and pressures during a bed operation: influence of body types, body positions, and knee positions. Wound Repair Regen 2009;17(6):789-96.

[4] Bernhardsson S, Lynch E, Dizon JM, et al. Advancing evidence-based practice in physical therapy settings: multinational perspectives on implementation strategies and interventions. Phys Ther 2017;97(1):51-60.

[5] Jack K, McLean SM, Moffett JK, et al. Barriers to treatment adherence in physiotherapy outpatient clinics: a systematic review. Man Ther 2010;15(3):220-8.

[6] McLean SM, Burton M, Bradley L, et al. Interventions for enhancing adherence with physiotherapy: a systematic review. Man Ther 2010;15(6):514-21.

[7] Braithwaite I. Lower limb immobility and venous thromboembolism risk: Investigating preventive measures 2016

[8] Morris PE. Moving our critically ill patients: mobility barriers and benefits. Crit Care Clin 2007;23(1):1-20.

[9] Huskisson EC, Hart FD. Joint disease: all the arthropathies. Elsevier 2013.

[10] Ampiah PK, Ahenkorah J, Karikari M. Patients' satisfaction with inpatient orthopedic physiotherapy services at a tertiary hospital in Ghana. J Patient Exp 2019;6(3):23846.

[11] Tokunaga M, Higashi T, Inoue R, et al. Comparison of Functional Independence Measure (FIM) gain between groups with extreme differences in FIM score at admission. Int J Phys Med Rehabil 2017;5(402):2.

[12] De Lima EL, Brito MJ, de Souza DM, et al. Cross-cultural adaptation and validation of the neonatal/infant braden Q risk assessment scale. J tissue viability 2016;25(1):5765.

[13] Harper S. Economic and social implications of aging societies. Science 2014;346(6209):587-91.

[14] Giusti GD, Peghetti A, Comisso I, et al. Early mobility, skin and pressure ulcer risk assessment. Nursing in Critical Care Setting 2018:137-53. 